\title{
ASPECTS REGARDING SHOCK WAVE MITIGATION THROUGH DIFFERENT MEDIA
}

\author{
Iuliana-Florina PANĂ* \\ ciortan.florina@yahoo.ro \\ Luminiţa-Cristina ALIL* \\ aegyssusatm@yahoo.com \\ Florin ILIE** \\ ilieflorinv@yahoo.com
}

\author{
* MILITARY TECHNICAL ACADEMY, BUCHAREST, ROMANIA, \\ **"NICOLAE BĂLCESCU" LAND FORCES ACADEMY, SIBIU, ROMANIA
}

\section{ABSTRACT}

The main application of underwater detonation since the Second World War is to destroy military ships. Nowadays, a lot of studies are performed in order to discover a controlled and safe application of shock waves through different media. The paper presents the results of a research on a bubble curtain behaviour subjected to shock waves generated by an underwater TNT blast. The main objective was to analyze the mitigation solution of underwater explosion effects by means of gas bubbles. Simulations using ANSYS AUTODYN and explicit dynamics procedures were performed on a 3D model, in order to better understand the physical process of formation and propagation of a shock wave in the biphasic medium which represents the purpose of many researchers. The numerical simulations were performed taking into account the interaction between a shock wave and the bubble curtain considering a random distribution in space and bubble dimensions.

\section{Keywords}

Simulation, biphasic medium, shock weave, explicit dynamics

\section{Introduction}

Between modern war, demolitions, medicine, geophysics and material sciences, one can remark a clear connection with the domain of generation, propagation and attenuation of shock waves. Even if we talk about shock waves in the context of terrorist attack or in positive applications, the complexity of this domain is still a challenge for the scientists. After initiation, the explosive material suffers a chemical reaction, usually detonation for case of second explosives like TNT, resulting in generation of a detonation wave, which represents a discontinuity surface, and formation of reaction products. The detonation wave propagates from the centre of the explosion to the surface of the gas sphere and transmits 
energy to the exterior medium. The gaseous products result in a sphere and they move with a certain speed.

\section{Material Models}

To model the behaviour of water, air and TNT further equations are to be solved in the 3D mitigation configuration. In AUTODYN, Century Dynamics Inc. (2003), air behaviour is characterized by ideal gas equation of state, as follows: [1]

$$
p=(\gamma-1) \rho e+p_{\text {shift }},
$$

where $p$ is pressure, $\gamma$ is adiabatic coefficient, $\rho$ is density, $p_{\text {shift }}$ is a small initial value of pressure and $e$ is internal energy given by:

$$
e=c_{v} T,
$$

with $c_{v}$ the specific heat.

The TNT equation of state is given by Jones-Wilkins-Lee (JWL) equation, as follows:

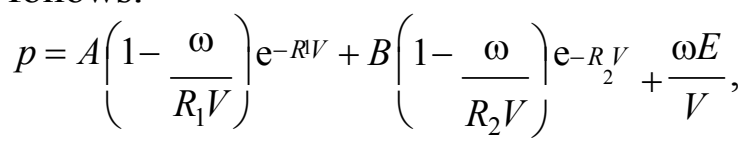

where $A, B, R_{1}, R_{2}, \omega$ are empirical constants that differ for each explosive material, $V$ is the relative volume, $E$ is the ratio between detonation energy and initial volume.

Regarding the water equation of state there is a polynomial form that characterizes the water behaviour:

$$
P=a_{1} \mu+a_{2} \mu^{2}+a_{3} \mu^{3}+\left(b_{0}+b_{1} \mu\right) \rho_{0} E,
$$

where $\mu$ is the compression parameter, $\rho_{0}$ is initial density and $a_{1}, a_{2}, a_{3}, b_{0} b_{1}$ are defined in AUTODYN's material library.

\section{Basic Formulation of Explicit Dynamics}

The basic equation solved by an explicit dynamic analysisexpress the conservation of mass, momentum and energy in Lagrange coordinates. These, together with a material model and a set of initial and boundary conditions, define the complete solution of the problem.

For Lagrange formulations, the mesh moves and distorts with the material. The density at any time can be determined from the current volume of the zone $V_{0}$ and its initial mass $\left(\rho_{0} V_{0}\right)[2]$ :

$$
\rho=\frac{\mathrm{m}}{\mathrm{V}}=\frac{\rho_{0} \mathrm{~V}_{0}}{\mathrm{~V}}
$$

Energy conservation is written as: [3]

$$
\begin{aligned}
\dot{e}= & \frac{1}{\rho}\left(\sigma_{x x} \dot{\varepsilon}_{x x}+\sigma_{y y} \dot{\varepsilon}_{y y}+\sigma_{z z} \dot{\varepsilon}_{z z}+\right. \\
& \left.2 \sigma_{x y} \dot{\varepsilon}_{x y}+2 \sigma_{y z} \dot{\varepsilon}_{y z}+2 \sigma_{z x}^{\dot{\varepsilon}}\right)
\end{aligned}
$$

For each time step, these equations are solved explicitly for each element in the model, based on input values at the end of the previous time step. The explicit dynamics solver uses a central difference time integration scheme - Leapfrog method. The advantages of using this method for time integration are:

- equations can be solved directly (explicitly);

- no convergence checks are needed since the equations are uncoupled;

- no inversion of the stiffness matrix is required.

\section{Numerical Simulation of Shock Wave Propagation in Biphasic Medium}

\subsection{Introduction}

Numerical simulations performed until now give information about gas bubbles evolution, reflection and transfer of shock wave, the temperature inside and outside the bubble.

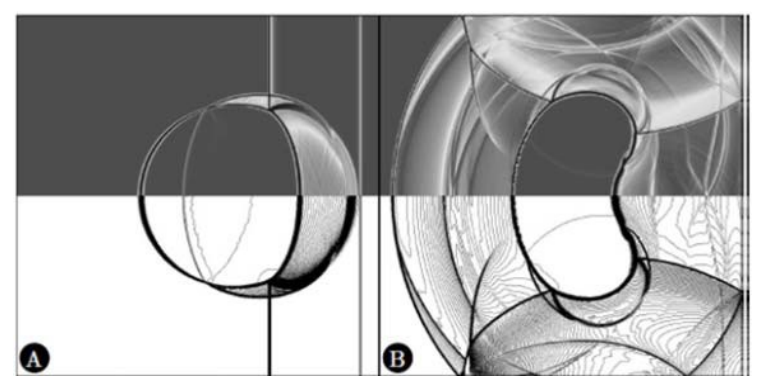




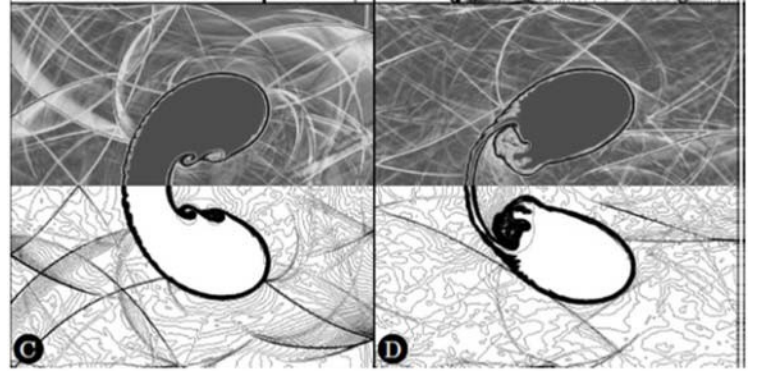

Figure no. 1 Bubble behaviour under shock wave [4]

Some studies go further to analyzing the influence of a boundary near the bubble and its oscillations effects. Interesting results are provided by Ding and Gracewski's [5] simulation, figure no. 1. They assigned water an artificial viscosity and obtained information concerning the interaction between a strong plane wave and cavitations. The same group of researchers extended their studies on bubble oscillations in a vessel [6]. They implemented the BEM method (Boundary Element Method) to analyze the flow in sanguine vessels. One of the primary articles where simulations are based on BEM method belongs to Klaseboer [7], where Bernoulli's equation is used to model the plane wave.
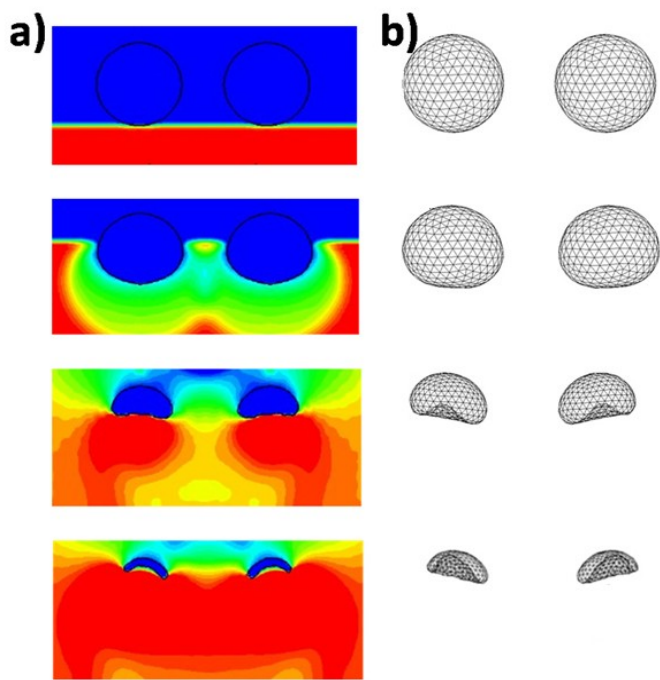

Figure no. 2 Numerical simulation of shock wave propagation in bubbly liquid with BEM method (right) and Euler solver (left) [8]
A strong shock wave with a $100 \mathrm{MPa}$ pick overpressure is modeled using two different simulation methods exemplified in figure 2. The succession of events is illustrated from the top to the bottom and the colour code represents the fluid velocity starting with the low values (blue) to the maximum ones (red).

\subsection{Case study}

The configuration studied from the numerical point of view starts with the 1D simulation of $50 \mathrm{~g}$ of TNT detonation, figure no. 3 .
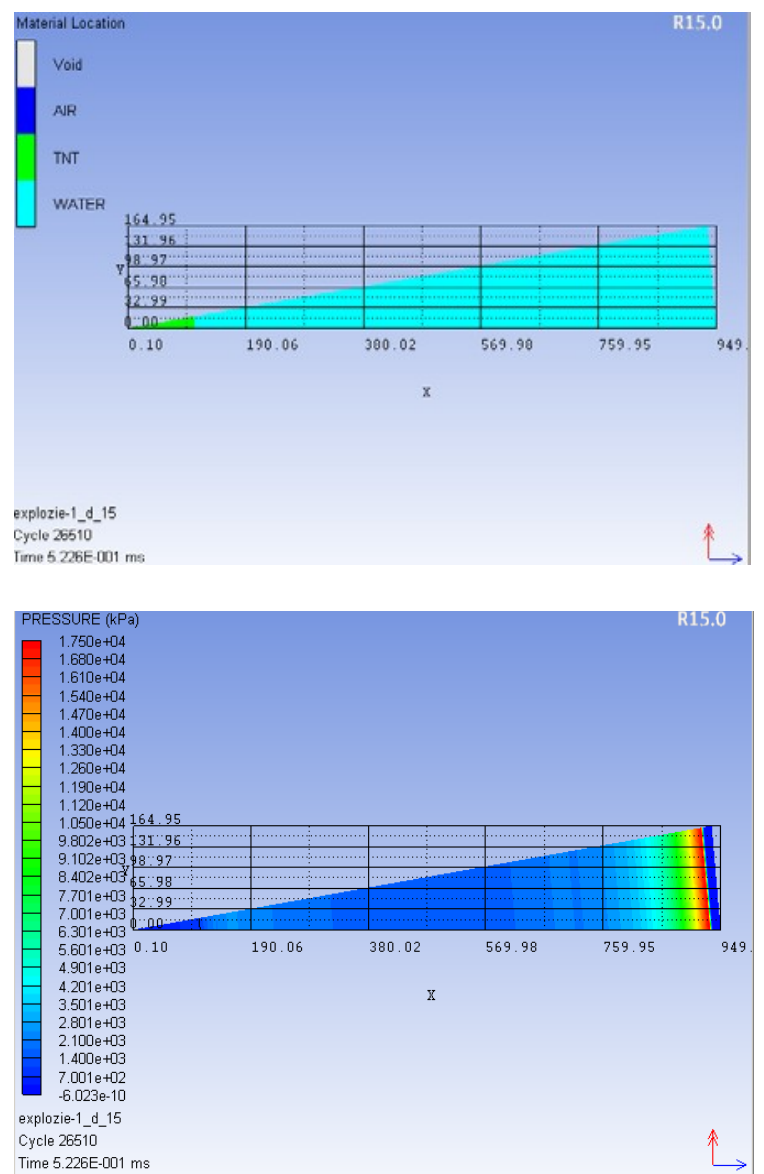

\section{Figure no. $31 \mathrm{D}$ blast simulation}

The main purpose of this simulation is to model the formation and propagation of shock wave in water before the interaction with bubbles as strict as possible and without much computational resources. 

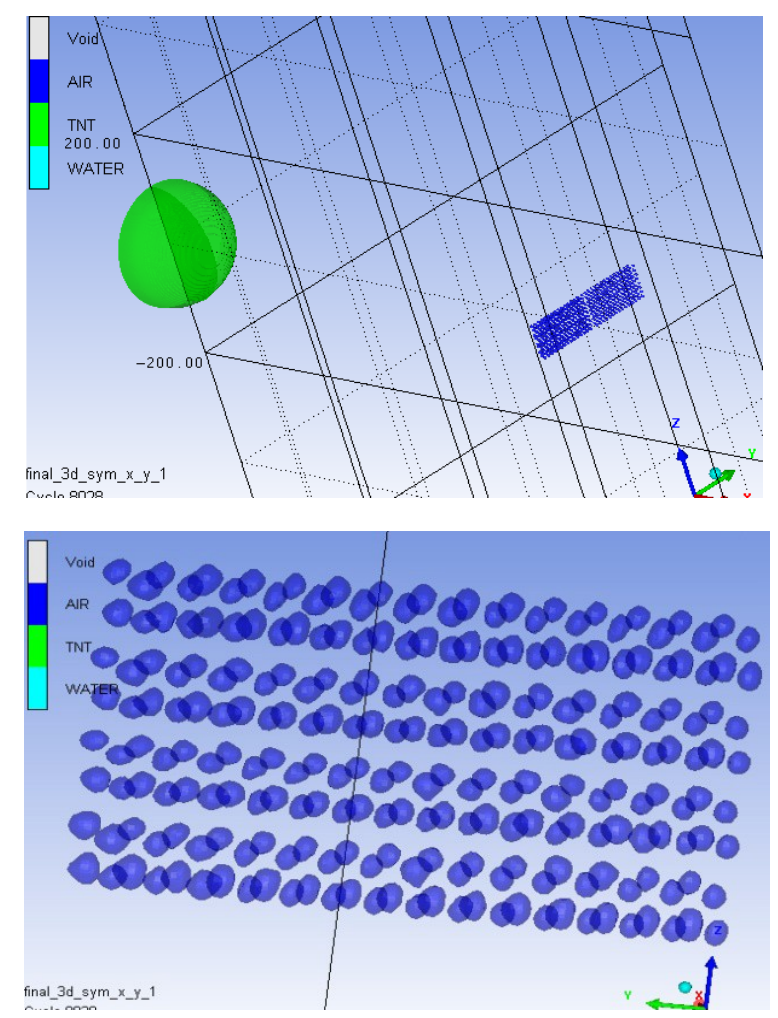

Figure no. 4 3D blast simulation

The results obtained from the 1D simulation were introduced in the 3D model using the "remap" option in AUTODYN. In figure no. 4 is presented the $3 \mathrm{D}$ mitigation configuration (left) and the bubble curtain (right). The biphasic medium was simulated taking into account the random distribution of bubble in space and the dimensions as it can be observed from figure no. 4. The model is symmetric with $x$ and $y$ and the initial bubble dimensions were designed according to theoretical approach detailed in the previous chapter.

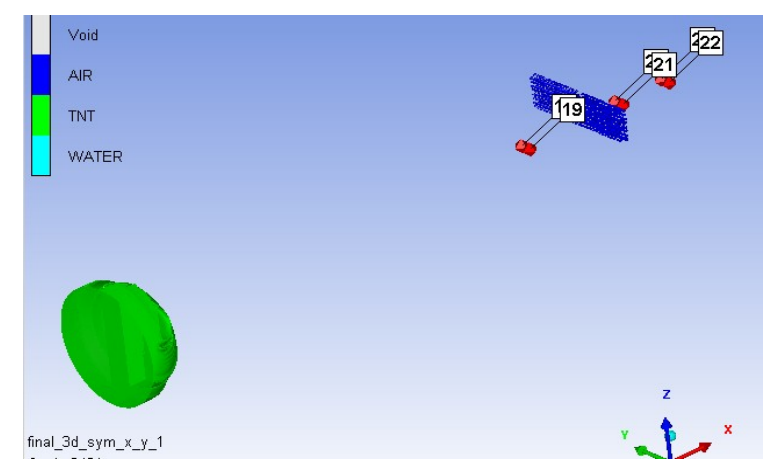

Figure no. 5 Gauge position in the mitigation configuration
The 3D model is conceived in "Euler multi-material" which permits the air fill of bubbles in a water cube. Firstly it was performed a simulation (1.8 $\mathrm{ms}$ long) only with the bubbles in the water with an initial velocity condition approximately equal to 5 $\mathrm{cm} / \mathrm{s}$. In figure no. 5 it is highlighted the position of the virtual sensors which will provide information regarding the pick overpressure before and after the interaction between blast wave and bubble curtain.

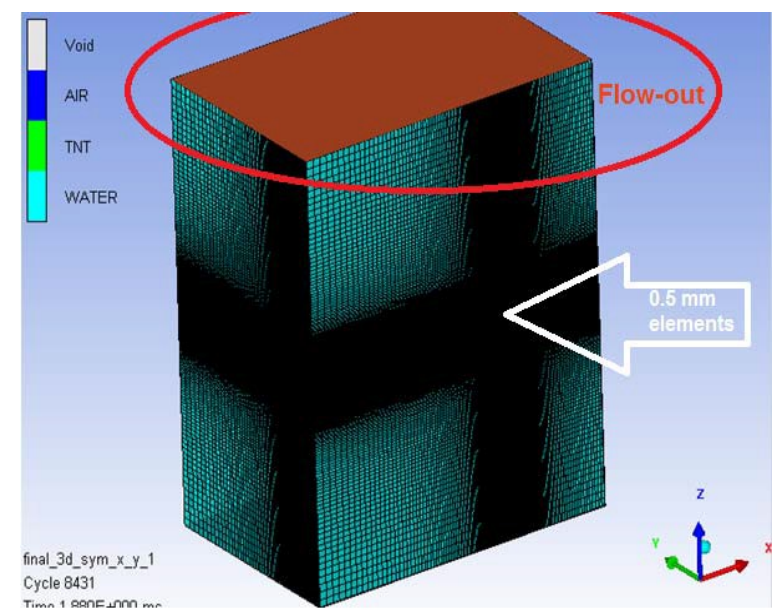

Figure no. 6 Boundary conditions and model mesh

The 3D model contains almost 17 millions elements and it is meshed as in the figure 6 . The "flow out" boundary condition is presented on the top of the model and the rest of the cub's walls are considered rigid.

\section{Conclusions and Results}

Because of the wave dispersion phenomena (the velocity of waves with different lengths varies with bubble pulsation in liquid) after its propagation in bubbly liquid, theoretical approach of biphasic mediums differs considerably from the single phase liquids. The presence of the gas bubbles in an incompressible liquid forms a mix which is able to sustain shock wave load.

The paper's purpose is to build a direction to modeling a biphasic medium, liquid-gas, as closed to real phenomena as possible in order to be able to simulate the 
mitigation of the shock wave effects in a complex and conclusive manner.

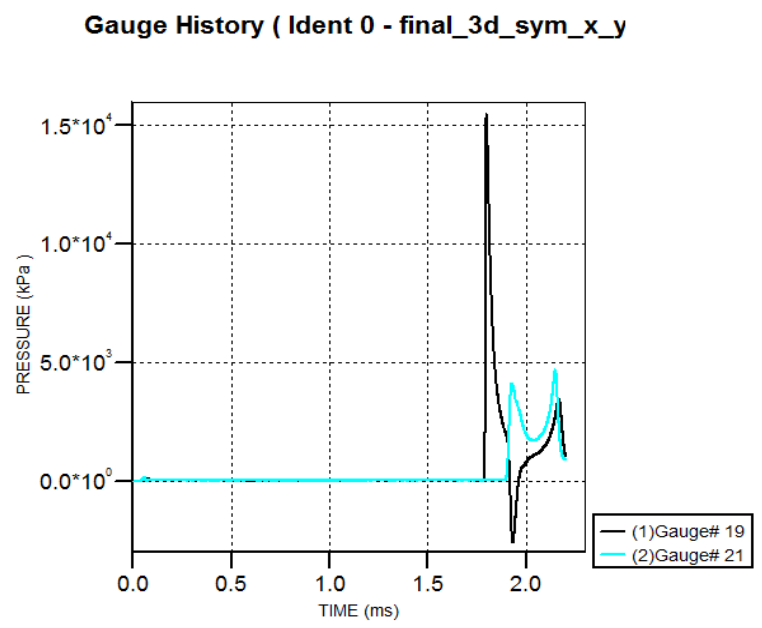

Figure no. 7 Pick overpressure before the bubble curtain (black) and after (blue)
Numerical approach is based on the following assumptions:

air is an ideal gas;

bubble collapse is neglected;

the bubble curtain is modeled as a reduced bubble wall configuration in front of the explosion.

The results provided by numerical analyze with ANSYS AUTODYN are materialized in figure no. 7 where one can observe the overpressure profile with time in two points placed before (black overpressure profile) and after (blue overpressure profile) the bubble curtain.

\section{Acknowledgment}

The paper was financially supported through the project "Horizon 2020 - Doctoral and Postdoctoral Studies: Promoting the National Interest through Excellence, Competitiveness and Responsibility in the Field of Romanian Fundamental and Applied Scientific Research", contact identification number POSDRU/159/1.5/S/140106. This project is co-financed by European Social Fund through Sectoral Operational Program for Human Resources Development 2007-2013. Investing in people!

\section{References}

1. AUTODYN User Manual Version 12.1.

2. Ibidem.

3. Ibidem.

4. $\quad$ Ding Z. and Gracewski S.M., "The Behaviour of a Gas Cavity Impacted by a Weak or Strong Shock Wave”, Journal of Fluid Mechanics, Vol. 309, (1996): 183-209.

5. Ibidem.

6. Miao, H., Gracewski, S.M. and Dalecki, D., "Ultrasonic excitation of a bubble inside a deformable tube: implications for ultrasonically induced hemorrhage", The Journal of the Acoustical Society of America., Vol 124, (2008).

7. Ding Z. and Gracewski S.M., cit.ed.

8. Klaseboe E., Turangan C., Fong S.W., Liu T.G., Hung K.C. and Khoo B.C., "Simulations of Pressure Pulse-Bubble Interaction Using Boundary element Method", Compter Methods in Applied Mechanics and Engineering, Vol. 195, (2006). 


\section{Bibliography}

AUTODYN User Manual Version 12.1.

Ding Z. and Gracewski S.M., "The Behaviour of a Gas Cavity Impacted by a Weak or Strong Shock Wave", Journal of Fluid Mechanics, Vol. 309, (1996): 183-209.

Klaseboe E., Turangan C., Fong S.W., Liu T.G., Hung K.C. and Khoo B.C., "Simulations of Pressure Pulse-Bubble Interaction Using Boundary element Method", Compter Methods in Applied Mechanics and Engineering, Vol. 195, (2006).

Miao, H., Gracewski, S.M. and Dalecki, D., "Ultrasonic excitation of a bubble inside a deformable tube: implications for ultrasonically induced hemorrhage", The Journal of the Acoustical Society of America, Vol 124, (2008). 